\title{
DESIGN OF LINEAR QUADRATIC REGULATOR (LQR) BASED ON GENETIC ALGORITHM FOR INVERTED PENDULUM
}

\author{
Tomáš Marada, Radomil Matoušek, Daniel Zuth \\ Brno University of Technology, Faculty of Mechanical Engineering \\ Institute of Automation and Computer Science \\ Technicka 2896/2, 616 69, Brno \\ Czech Republic \\ marada@fme.vutbr.cz
}

\begin{abstract}
One of the crucial problems in the dynamics and automatic control theory is balancing of an inverted pendulum robot by moving a cart along a horizontal path. This task is often used as a benchmark for different method comparison. In the practical use of the LQR method, the key problem is how to choose weight matrices $Q$ and $R$ correctly. To obtain satisfying results the experiments should be repeated many times with different parameters of weight matrices. These LQR parameters can be tuned by a Genetic Algorithm (GA) technique for getting better results. In our paper, the $L Q R$ parameters weight matrices $Q$ and $R$ which were tuned using the Genetic Algorithm. The simulations of the control problem are designed using MATLAB script code and MATLAB Simulink on an inverted pendulum model. The results show that the Genetic Algorithm is suitable for tuning the parameters to give an optimal response. The control problem of the inverted pendulum was solved successfully.
\end{abstract}

Keywords: Inverted pendulum, Linear Quadratic regulator (LQR), Genetic algorithm.

\section{Introduction}

The motivation for the study of the inverted pendulum was a requirement to design a control system to attitude control of a rocket booster at take-off [1]. During the initial phase, the space rocket booster is extremely unstable. An inverted pendulum, like a rocket, requires constant correction of its position to remain upright. Another real application that has the same idea as the inverted pendulum is a two-wheeled self-balancing battery-powered electric Segway PT, invented by Dean Kamen [3]. Noteworthy examples can be automated aircraft landing systems, aircraft stabilization in turbulent flow, stabilization of cabins in ships etc.

One of the crucial problems in the dynamics and automatic control theory is balancing of an unstable nonlinear inverted pendulum. An inverted pendulum is mounted on a motorized cart which can move along a horizontal path [1], [2]. An inverted pendulum is often used as a traditional benchmark for evaluating various control strategies. For example PID controllers [4], Linear Quadratic Regulators [5], neural networks, genetic algorithms [6] and fuzzy control methods. The aim is to swing up the inverted pendulum from the stable vertical down position to the unstable vertical up position and to keep the pendulum in vertical up position. The swing up process uses cart movements to add energy to the pendulum. The cart is driven by a force from a servo motor. It is also important to reduce cart movements amplitude. The swing up method puts the pendulum to the unstable vertical up position with small angular velocity. The inverted pendulum stabilization can be accomplished via several methods. In this paper LQR method (Linear Quadratic regulator) is used to generate the signal to control the servo motor. In the practical use of the LQR method, the key problem is how to choose weight matrices $\mathrm{Q}$ and $\mathrm{R}$ correctly. To obtain the satisfying results the experiments should be repeated many times with different parameters of weight matrices. These LQR parameters can be tuned by a Genetic Algorithm (GA) [6] technique for getting better results. In our paper, the LQR parameters weight matrices Q and $\mathrm{R}$ were tuned using the Genetic Algorithm. The simulations of the control problem are designed by means of MATLAB script code and MATLAB Simulink on an inverted pendulum model. 


\section{Mathematical Model of the Inverted Pendulum}

We consider a two-dimensional problem in our case only. The pendulum is limited to move in the vertical plane shown in Fig. 1. The cart with pendulum can move to the right or to the left on the rail freely. The pendulum is connected to the center of the cart and can rotate around the pivot. For this system, the control input is the force $F$ that moves the cart horizontally. The first output is the pendulum angular position $\theta$ and the second is the cart horizontal position $x$.

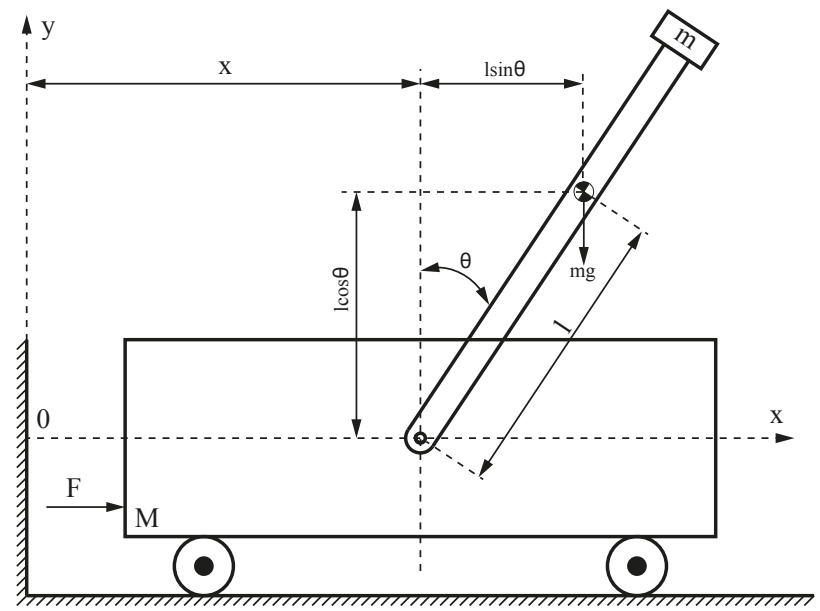

Figure 1: Inverted pendulum schematic

Table 1: Inverted pendulum parameters

\begin{tabular}{cll}
\hline Symbol & Parameter & Value \\
\hline$F$ & force applied to the cart & $\mathrm{kg} \cdot \mathrm{m} / \mathrm{s}^{2}$ \\
$M$ & mass of the cart & $2.5 \mathrm{~kg}$ \\
$m$ & mass of the pendulum & $0.25 \mathrm{~kg}$ \\
$g$ & gravity & $9.81 \mathrm{~m} / \mathrm{s}^{2}$ \\
$I$ & mass moment of inertia of the pendulum & $0.0102 \mathrm{~kg} \cdot \mathrm{m}^{2}$ \\
$l$ & length to pendulum center of mass & $0.35 \mathrm{~m}$ \\
$B$ & the coefficient of viscous friction for the cart & $0.5 \mathrm{~N} / \mathrm{m} / \mathrm{sec}$ \\
$b$ & the viscous friction of the pendulum on the axis of rotation & $0.0004 \mathrm{~N} / \mathrm{m} / \mathrm{sec}$ \\
$\theta$ & pendulum angle from vertical & degree \\
$x$ & cart horizontal position & $\mathrm{m}$ \\
\hline
\end{tabular}

For numerical simulations of the inverted pendulum in MATLAB Simulink, nonlinear equations are required. These nonlinear dynamic system equations were derived in the paper [9] in detail.

$$
\begin{aligned}
& (m+M) \ddot{x}+b_{1} \dot{x}+m l \ddot{\theta} \cos \theta-m l \dot{\theta}^{2} \sin \theta=F \\
& \ddot{\theta}\left(I+m l^{2}\right)+m l \ddot{x} \cos \theta-m g l \sin \theta+b_{2} \dot{\theta}=0
\end{aligned}
$$

Where (1) is the nonlinear motion equation of the cart and (2) is the nonlinear motion equation of the pendulum. These equations need to be linearized in order to represent the system in state space form. Linearization of motion equations is performed in an upright balanced position where $\theta=0$ assuming small deviations from equilibrium. This assumption can be regarded as fulfilled since the control is realized for the pendulum displacement of less than 20 degrees from the vertical upright position. For small angular displacements we can write $\cos (\theta) \approx 1, \sin (\theta) \approx 0$ and $\dot{\theta}^{2}=0$. By substituting into the nonlinear motion equations for the position of the carriage (1) and rotation of the pendulum (2) we obtain two linearized equations of motion (3), (4) for the upper unstable position $\theta=0$.

$$
\begin{gathered}
(m+M) \ddot{x}+b_{1} \dot{x}+m l \ddot{\theta}=F \\
\ddot{\theta}\left(I+m l^{2}\right)+m l \ddot{x}-m g l \theta+b_{2} \dot{\theta}=0
\end{gathered}
$$




\section{Inverted pendulum state model}

For the linear state feedback control of the inverted pendulum it is needed to convert the motion equations into a continuous system state space model of the form (5).

$$
\begin{aligned}
\dot{x}(t) & =A x(t)+B u(t) \\
y(t) & =C x(t)+D u(t)
\end{aligned}
$$

The state vector is chosen $[x, \theta, \dot{x}, \dot{\theta}]^{T}$, where the cart position is $x$, the deflection angle of the pendulum from the upper equilibrium position is $\theta$, the cart speed is $\dot{x}$ and the angular velocity of the pendulum is $\dot{\theta}$. We want to make (3) and (4) more convenient for the state space representation by expressing $\ddot{x}$ and $\ddot{\theta}$ in the following way:

$$
\begin{aligned}
& \ddot{x}=\frac{1}{(m+M) I+M m l^{2}}\left\{-b_{1}\left(I+m l^{2}\right) \dot{x}-m^{2} l^{2} g \theta+b_{2} m l \dot{\theta}+\left(I+m l^{2}\right) F\right\} \\
& \ddot{\theta}=\frac{1}{(m+M) I+M m l^{2}}\left\{m l b_{1} \dot{x}+m \lg (m+M) \theta-b_{2}(m+M) \dot{\theta}-m l F\right\}
\end{aligned}
$$

The system in state space form is then (8) and (9):

$$
\begin{gathered}
\dot{x}=\left[\begin{array}{cccc}
0 & 0 & 1 & 0 \\
0 & 0 & 0 & 1 \\
0 & \frac{-m^{2} l^{2} g}{(m+M) I+M m l^{2}} & \frac{-b_{1}\left(I+m l^{2}\right)}{(m+M) I+M m l^{2}} & \frac{b_{2} m l}{(m+M) I+M m l^{2}} \\
0 & \frac{m \lg (m+M)}{(m+M) I+M m l^{2}} & \frac{m l b_{1}}{(m+M) I+M m l^{2}} & \frac{-b_{2}(m+M)}{(m+M) I+M m l^{2}}
\end{array}\right]\left[\begin{array}{c}
x \\
\theta \\
\dot{x} \\
\dot{\theta}
\end{array}\right]+\left[\begin{array}{c}
0 \\
0 \\
\frac{I+m l^{2}}{(m+M) I+M m l^{2}} \\
\frac{-m l}{(m+M) I+M m l^{2}}
\end{array}\right] u \\
y=\left[\begin{array}{c}
x \\
\theta
\end{array}\right]=\left[\begin{array}{llll}
1 & 0 & 0 & 0 \\
0 & 1 & 0 & 0
\end{array}\right]\left[\begin{array}{c}
x \\
\theta \\
\dot{x} \\
\dot{\theta}
\end{array}\right]+\left[\begin{array}{c}
0 \\
0
\end{array}\right] u
\end{gathered}
$$

The obtained state matrices $A, B, C, D$ for $\theta=0$ have the form:

$$
\begin{aligned}
& A=\left[\begin{array}{cccc}
0 & 0 & 1 & 0 \\
0 & 0 & 0 & 1 \\
0 & \frac{-m^{2} l^{2} g}{(m+M) I+M m l^{2}} & \frac{-b_{1}\left(I+m l^{2}\right)}{(m+M) I+M m l^{2}} & \frac{b_{2} m l}{(m+M) I+M m l^{2}} \\
0 & \frac{m \lg (m+M)}{(m+M) I+M m l^{2}} & \frac{m l b_{1}}{(m+M) I+M m l^{2}} & \frac{-b_{2}(m+M)}{(m+M) I+M m l^{2}}
\end{array}\right]=\left[\begin{array}{cccc}
0 & 0 & 1 & 0 \\
0 & 0 & 0 & 1 \\
0 & -0.7180 & -0.1951 & 0.0003 \\
0 & 22.5645 & 0.4182 & -0.0105
\end{array}\right] \\
& B=\left[\begin{array}{c}
0 \\
0 \\
\frac{I+m l^{2}}{(m+M) I+M m l^{2}} \\
\frac{-m l}{(m+M) I+M m l^{2}}
\end{array}\right]=\left[\begin{array}{c}
0 \\
0 \\
0.3902 \\
-0.8364
\end{array}\right] \\
& C=\left[\begin{array}{llll}
1 & 0 & 0 & 0 \\
0 & 1 & 0 & 0
\end{array}\right] \\
& D=\left[\begin{array}{l}
0 \\
0
\end{array}\right]
\end{aligned}
$$

State matrices obtained by the analytical method are the same as the state matrices obtained in MATLAB by typing the command: $[\mathrm{A}, \mathrm{B}, \mathrm{C}, \mathrm{D}]$ = linmod(model); where the model is previously created nonlinear inverted pendulum model created in MATLAB Simulink. This process is used for verifying the accuracy of analytical calculations.

\section{Genetic Algorithm (GA)}

Genetic Algorithms (GA) [6], [9] are search methods inspired by the laws of natural selection and genetics. Genetic Algorithm can be applied to search, optimization, machine learning tasks and various problems in control systems. GA starts with an initial population containing a number of chromosomes. Each one represents a potential problem solution or a better solution to a given optimization problem. Performance is evaluated by a fitness function. Each new generation is generated by applying three genetic operators Selection, Crossover and 
Mutation on old generation. Then good genes that lead to better fitness function in the new generation have more chance to survive. After some generations, the optimal solution will be reached. Flowchart of the genetic algorithm for LQR tuning is shown in Fig. 2. The genetic algorithm goal is to select LQR weight matrices, that will give a minimum fitness value, automatically. This control method is called GALQR. The genetic algorithm parameters were set to values shown in Table 3.

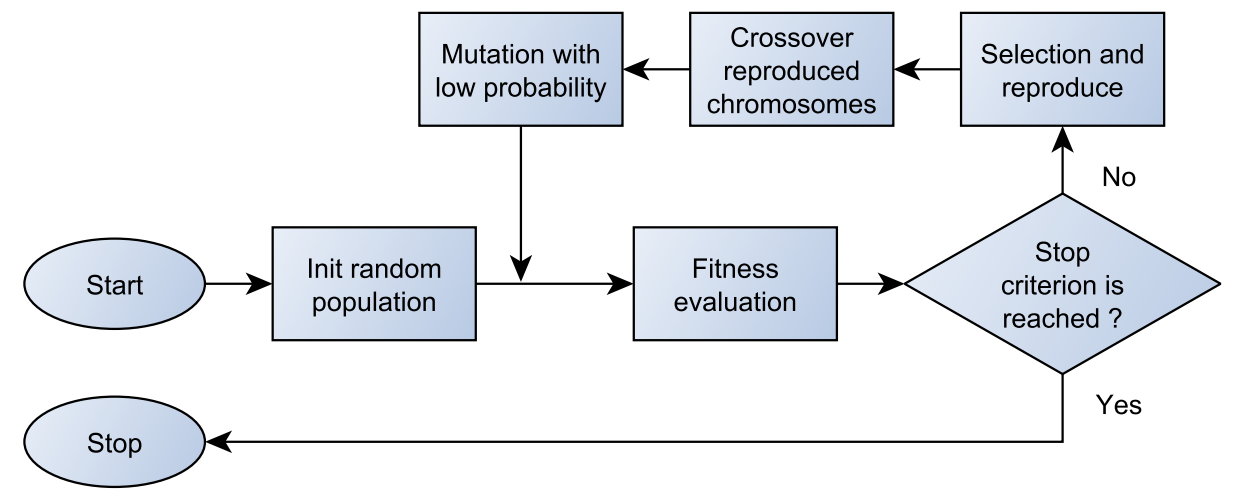

Figure 2: Flowchart of genetic algorithm for LQR tuning

\section{$5 \quad$ Fitness Function}

A definition of the fitness function, that is used to evaluate the fitness of each chromosome, is the most important task in applying the genetic algorithm. Each set of LQR parameters is passed to the objective function in order to compute fitness value. A good LQR controller must make the closed loop system as fast as possible, as stable as possible and with the lowest control effort as possible. Three indexes for the mentioned requirements were defined.

The stability index (14) is related to the real parts of closed-loop poles and is defined as follows.

$$
S I=-\frac{1}{\max _{i}\left(\operatorname{Re}\left\{\lambda_{i}\right\}\right)}
$$

The settling time index (15) measures the minimum time that the response reaches absolute error of $2 \%$,

$$
S T=\min \left\{t:\left|\frac{y(t)-y_{d}(t)}{y_{d}(t)}\right| \leq 0.02\right\}
$$

where $y(t)$ is the time response and $y_{d}(t)$ is desired output. If a desired value of the output signal is zero, then settling time is defined as follows (16).

$$
S T=\min \left\{t:|y(t)| \leq \frac{\max _{t} y(t)}{100} \cdot 2\right\}
$$

The maximum control effort index (17) is constrained by the limited capabilities of actuators. The control task must be achieved with minimum control effort. Higher saturation level actuators are more expensive.

$$
u_{\max }=\max _{t}|u(t)|
$$

The values of weight matrices $Q$ and $R$ were found using three indexes above. This problem is a multiobjective optimization problem and the fitness function was defined with the following equation (18).

$$
\text { fitness }=\alpha \cdot S I+\beta \cdot S T+\gamma \cdot u_{\max }
$$

Where $\alpha, \beta, \gamma$ represent the weight coefficients. Weight $\alpha=10$, weight $\beta=5$ and weight $\gamma=1$. 


\section{Experiments and Results}

In this section, the proposed method is applied to the inverted pendulum model introduced in section 3 . The numerical simulations were performed using MATLAB Simulink. The simulation time was set to 10 seconds, with step size of 0.01 seconds. The initial cart position was set to $0.1 \mathrm{~m}$ and the initial pendulum angle was set to $-0.02 \mathrm{rad}$. The required cart position was set to $0 \mathrm{~m}$ and the required pendulum angle was set to 0 rad. The control input $u$ was limited for all cases in ranges $[-1,1] \mathrm{N}$.

Two control schemes were implemented to control dynamical system of inverted pendulum:

- PID control method with cart position and pendulum angle PID controllers,

- GALQR control method where weight matrices were tuned with the Genetic Algorithm (similar as [12]).

The disturbance input to the system was added with band limited white noise block. The parameters of the disturbance input were set: noise power $=0.001$, sample time $=0.01$, seed $=23341$.

PID controllers were tuned using a trial and error method and the responses achieved to be optimal are observed. In Table 2 the tuned PID controller's parameters for cases of without and with disturbance input are given.

Table 2: PID controller parameters

\begin{tabular}{ccccc}
\hline PID & \multicolumn{2}{c}{ Without disturbance } & \multicolumn{2}{c}{ With disturbance } \\
parameter & Cart & Pendulum & Cart & Pendulum \\
\hline$K_{p}$ & -1 & -40 & -1.25 & -40 \\
$K_{i}$ & 0 & 0 & 0 & 0 \\
$K_{d}$ & -3 & -8 & -3.6 & -8 \\
\hline
\end{tabular}

The MATLAB Simulink models for inverted pendulum system control using PID control method without and with disturbance input are shown in Fig. 3 and Fig. 4. The cart position $x$ and the pendulum angle $\theta$ are used for the measurement only.

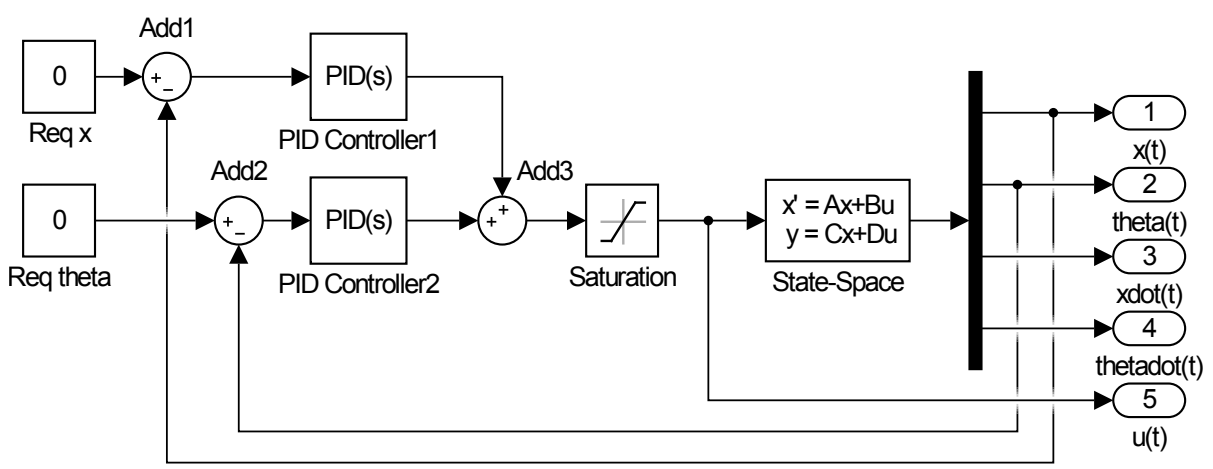

Figure 3: PID control of inverted pendulum system without disturbance input

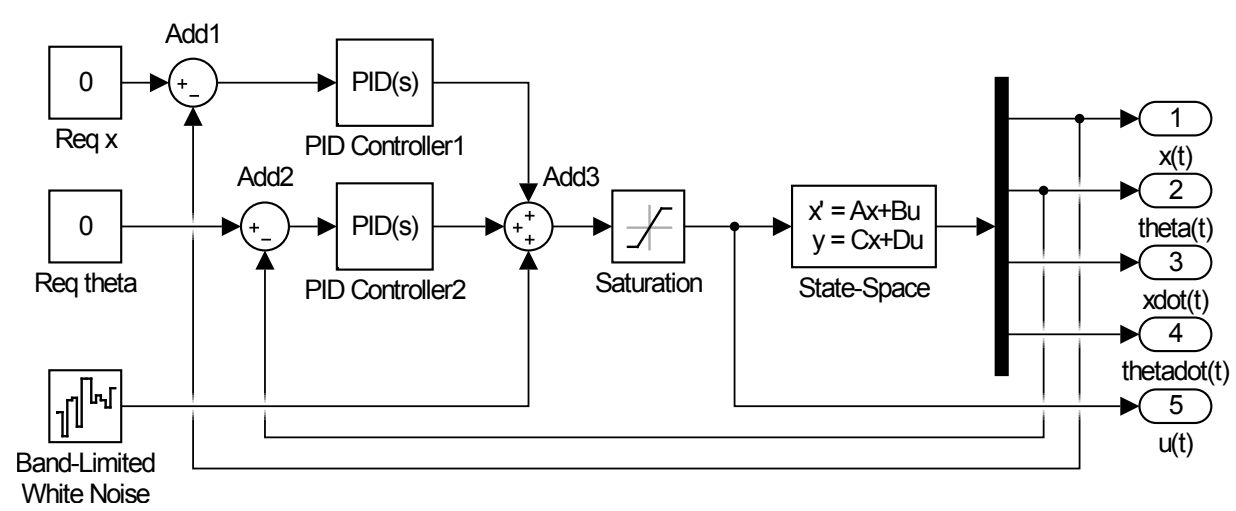

Figure 4: PID control of inverted pendulum system with disturbance input 
The simulation results for PID control of the inverted pendulum system without and with disturbance signal are shown in Fig. 5. The responses of cart position $x$, cart velocity $\dot{x}$, pendulum angle $\theta$, angular velocity $\dot{\theta}$ and action value $u$ are plotted. The inverted pendulum was stabilized in the vertical upright position after two overshoots and two small undershoots for the case of control without disturbance input. The inverted pendulum was also stabilized in the vertical upright position with several minor oscillations for the case of control with disturbance input. The angular velocity approached $0 \mathrm{rad} / \mathrm{s}$ for the case of control without disturbance input during 8.7 seconds and it oscillated by approximately $+/-0.05 \mathrm{rad} / \mathrm{s}$ for the case of control with disturbance input. The required position of the cart was reached quickly and smoothly in approximately 7.3 seconds for both cases. The cart velocity reached zero for the case of control without disturbance input and it oscillated very near to zero for the case of control with disturbance input. Action value was bound in the range [-1,1] for both cases. These simulation results showed robustness and effectiveness of the PID control.
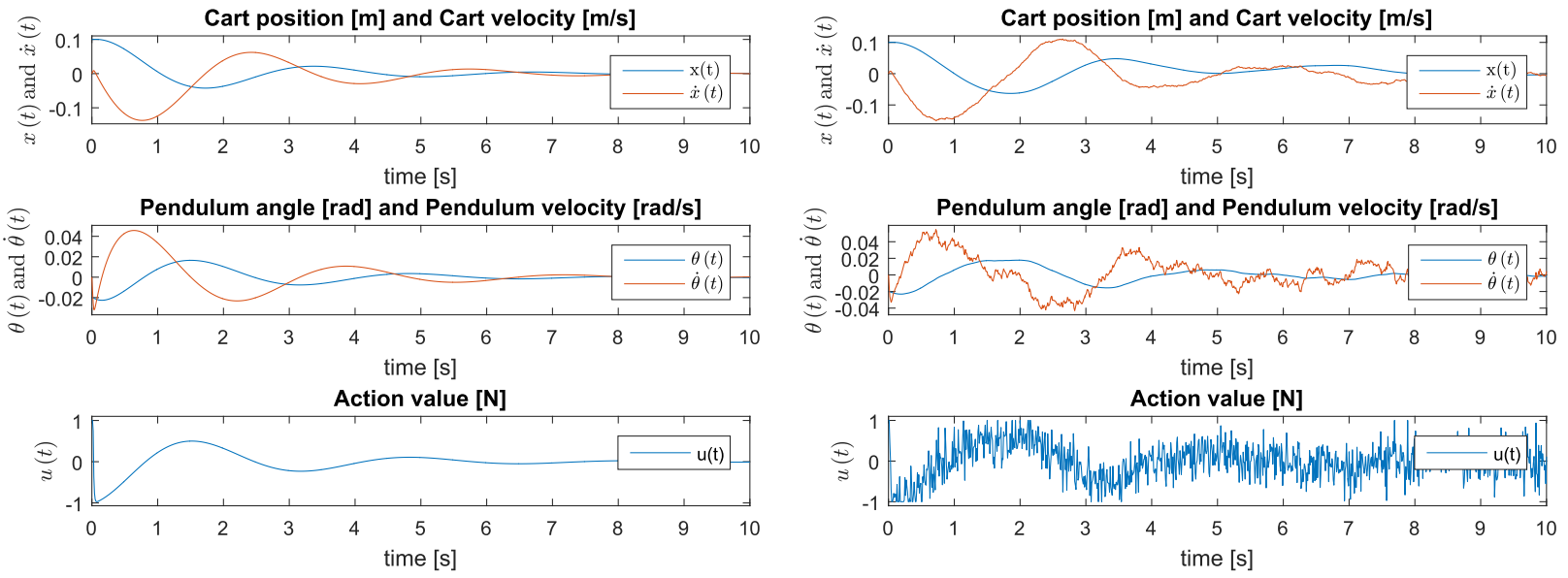

Figure 5: Responses of inverted pendulum system with PID control without and with disturbance signal

In the next part of the experiments the GALQR method was applied to the inverted pendulum control. To tune the LQR gain, the GA parameters were set to values shown in Table 3. That parameters were chosen based on [7].

Table 3: Specifications of the GA

\begin{tabular}{lc}
\hline GA parameter & Value \\
\hline Population size & 100 \\
Crossover rate & 0.8 \\
Generations number & 400 \\
\hline
\end{tabular}

The weight matrices were set using genetic algorithms. The optimal values of tuned weight matrix are (19) and the state feedback gain is (20).

$$
\begin{aligned}
& Q=\left[\begin{array}{cccc}
1000 & 0 & 0 & 0 \\
0 & 60.3005 & 0 & 0 \\
0 & 0 & 11.3453 & 0 \\
0 & 0 & 0 & 0.0083
\end{array}\right], \quad R=[1] \\
& K=\left[\begin{array}{cccc}
-31.6228 & -140.0440 & -27.7129 & -30.0572
\end{array}\right]
\end{aligned}
$$

The MATLAB Simulink models for the inverted pendulum system control using GALQR control method without and with disturbance input are shown in Fig. 6 and Fig. 7. All the states of the system $x, \dot{x}, \theta, \dot{\theta}$ are used in the GALQR.

The simulation results for GALQR control of the inverted pendulum system without and with disturbance signal are shown in Fig. 8. The responses of cart position $x$, cart velocity $\dot{x}$, angle $\theta$, angular velocity $\dot{\theta}$ and action value $u$ are plotted. The inverted pendulum was stabilized in the vertical upright position after one small overshoot for the case of control without disturbance input and the inverted pendulum was also stabilized in the vertical upright position with several minor oscillations for the case of control with disturbance input. The angular velocity approached $0 \mathrm{rad} / \mathrm{s}$ for the case of control without disturbance input during 2.1 seconds and it oscillated by approximately +/- $0.02 \mathrm{rad} / \mathrm{s}$ for the case of control with disturbance input. The required position 


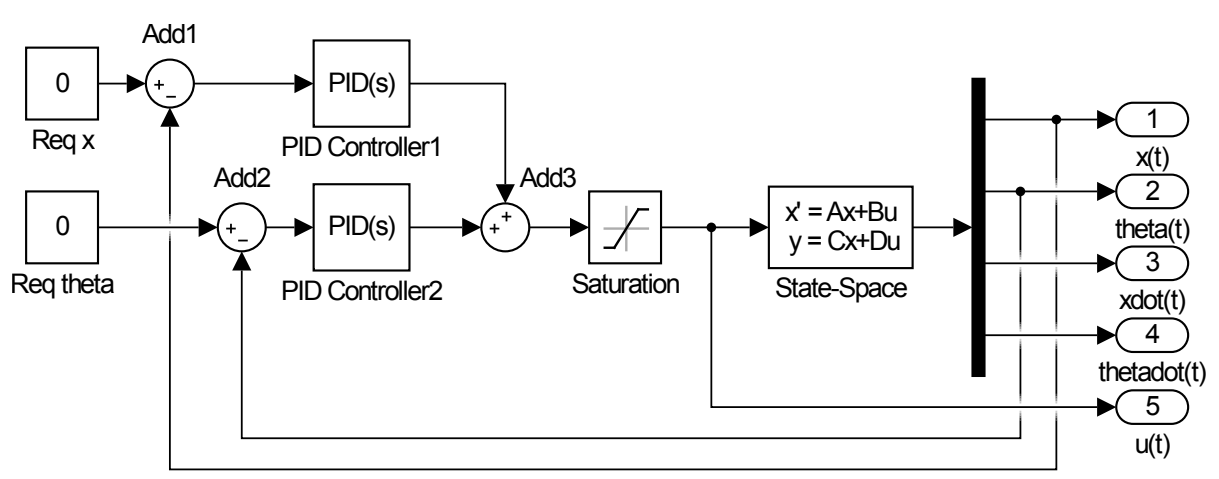

Figure 6: GALQR control of inverted pendulum system without disturbance input

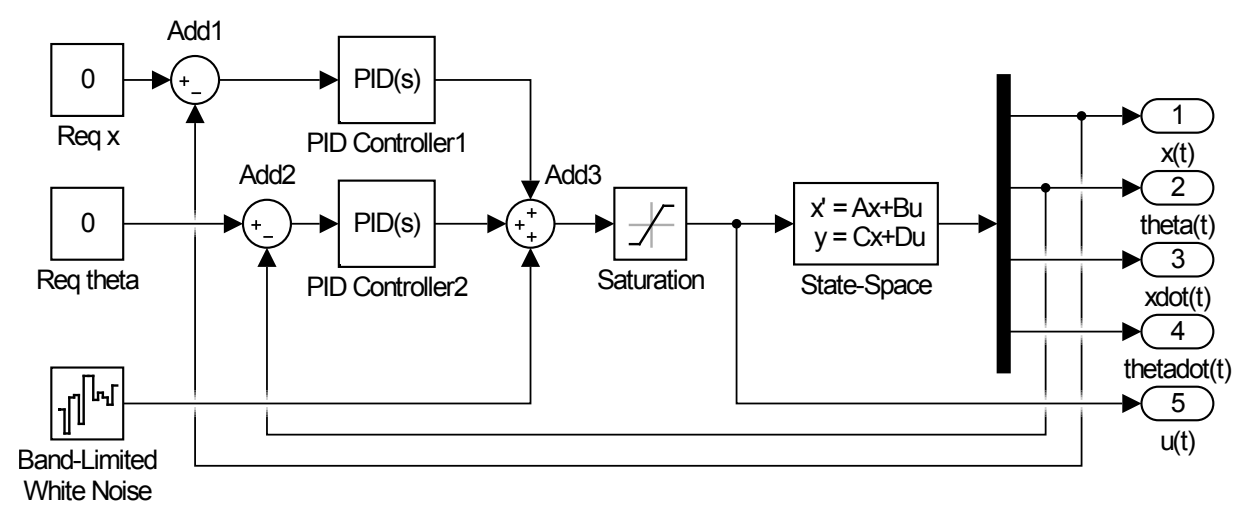

Figure 7: GALQR control of inverted pendulum system with disturbance input

of the cart was reached quickly and smoothly in approximately 1.5 seconds for both cases. The cart velocity reached zero for the case of control without disturbance input and it oscillated very near to zero for the case of control with disturbance input. Action value was bound in the range $[-1,0.5]$ for the case of control without disturbance input and in the range $[-1,1]$ for the case of control with disturbance input. These simulation results showed robustness and effectiveness of the GALQR control.
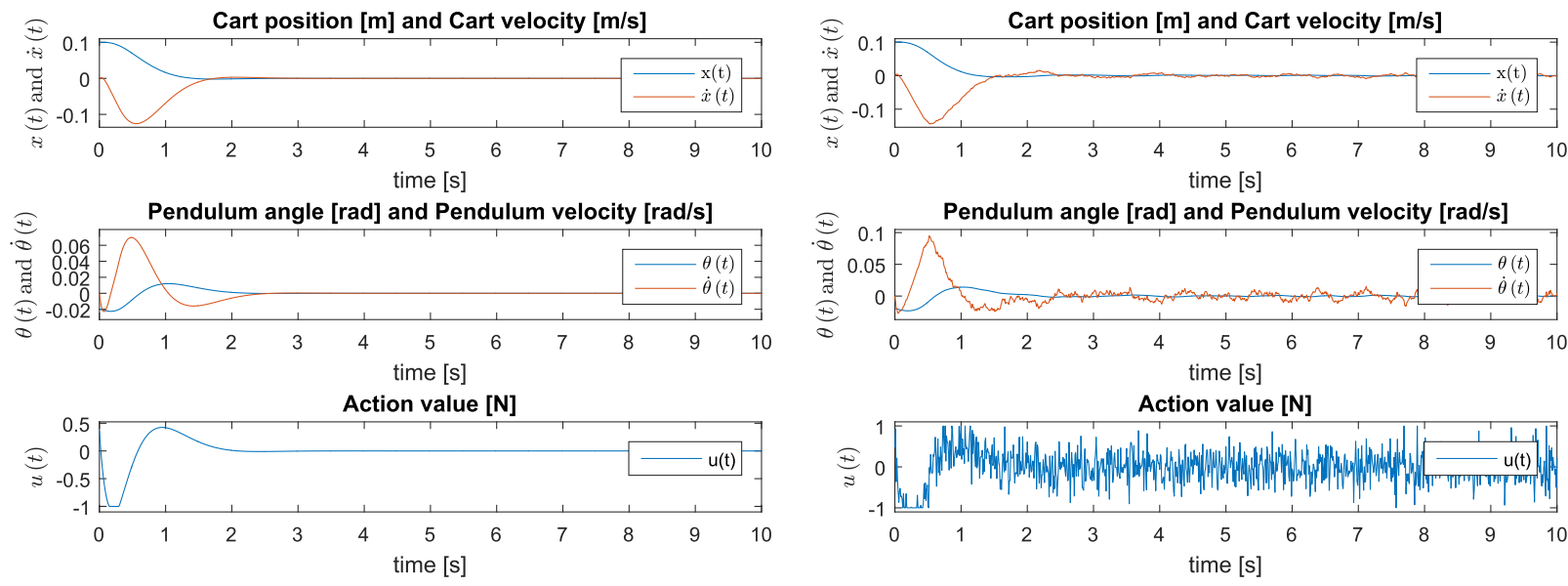

Figure 8: Responses of inverted pendulum system with GALQR control without and with disturbance signal

The performance of the used methods was observed in terms of Rising time, Settling time, ITAE criterion of the response and Fitness. The optimization results for the cart position and the pendulum angle are shown in Table 4 and Table 5. 
Table 4: Optimization results for cart position

\begin{tabular}{ccccc}
\hline Algorithm & Rise time & Settling time & ITAE & Fitness \\
\hline PID without disturbance & 0.6532 & 7.3333 & 0.2824 & - \\
PID with disturbance & 0.6170 & - & 0.7515 & - \\
GALQR without disturbance & 0.7964 & 1.3177 & 0.0303 & 21.6764 \\
GALQR with disturbance & 0.7109 & 2.0740 & 0.0608 & 64.6628 \\
\hline
\end{tabular}

Table 5: Optimization results for pendulum angle

\begin{tabular}{ccccc}
\hline Algorithm & Rise time & Settling time & ITAE & Fitness \\
\hline PID without disturbance & 0.3700 & 8.6513 & 0.0940 & - \\
PID with disturbance & 0.3460 & - & 0.1826 & - \\
GALQR without disturbance & 0.2431 & 2.1421 & 0.0148 & 21.6764 \\
GALQR with disturbance & 0.1985 & 9.8681 & 0.0414 & 64.6628 \\
\hline
\end{tabular}

\section{Conclusion}

In this paper, the problem of automatic finding of weight matrices for the LQR controller was formulated as an optimization problem. To solve this optimization problem the Genetic Algorithm (GA) was used. This method was applied for the inverted pendulum problem control. The MATLAB Simulink linearized model of the inverted pendulum system was developed for simulations and performance analysis of the control schemes. The obtained results from GALQR algorithm were compared with PID control algorithm. With respect to rise time, settling time, ITAE criterion and maximum control effort GALQR controller has better performance index than PID controller.

Acknowledgement: The work of the second author was supported by the Czech Science Foundation [1608549S] and by the Ministry of Education, Youth and Sports of the Czech Republic under the project CEITEC 2020 [LQ1601].

\section{References}

[1] Messner, B., Tilbury D.: Inverted Pendulum: System Modelling, http://ctms.engin.umich.edu/CTMS, [Online; accessed 25-April-2017]

[2] Andrew, K.S.: Standup and Stabilization of the Inverted Pendulum, Massachusetts Institute of Technology (1999)

[3] History of the Segway PT (Personal Transporter), http://www.isegway.cz/prague-segway-article/historyof-the-segway-pt, [Online; accessed 25-April-2017]

[4] Astrom, K.J., Murray, R.: Feedback systems: An Introduction for Scientists and Engineers, V2.10b, Princeton University Press (2009)

[5] Norman, S. N.: Control Systems Engineering, 6th edition, John Wiley \& Sons (2011)

[6] Mitchel, M.: An Introduction to Genetic Algorithms, fifth printing, MIT Press, Cambridge, Massachusetts (1999)

[7] MathWorks documentation, Genetic Algorithm, Genetic algorithm solver for mixed-integer or continuousvariable optimization, constrained or unconstrained, http://www.mathworks.com/help/gads/geneticalgorithm.html (2016), [Online; accessed 15-April-2016]

[8] MathWorks documentation, stepinfo, Rise time, settling time and other step response characteristics, http://www.mathworks.com/help/control/ref/stepinfo.html (2016), [Online; accessed 15-April-2016]

[9] Marada, T.: PID Controler Parameters Settings Based on Genetic Algorithm for Inverted Pendulum Purpose Stabilization. Mendel Journal series, In Mendel 2016, vol. 22, pp. 31-38. ISSN: 1803-3814 (2016)

[10] Zuth, D.: Using HIL Simulation and Genetic Algorithms for Controller Tuning. Mendel Journal series, In Mendel 2016, vol. 22, pp. 25-20. ISSN: 1803-3814 (2016)

[11] Šabartová, Z., and Popela, P.: Beam design optimization model with FEM based constraints. 18th International Conference on Soft Computing, MENDEL 2012; Brno; Czech Republic, (2012)

[12] Matousek, R., Lang, S., Minar, P., Pivonka, P.: Evolutionary Design of Polynomial Controller. An international Journal of Science, Engineering and Technology, World Academy of Science Engineering and Technology, Vol. 59, pp. 639-644. ISSN: 2010-376X (2011) 